\title{
Active Tremor Compensation in Microsurgery
}

\author{
W. T. Ang, P. K. Pradeep, and C. N. Riviere \\ The Robotics Institute, Carnegie Mellon University, Pittsburgh, PA, USA
}

\begin{abstract}
This paper presents the development of an intelligent microsurgical instrument to perform real-time tremor compensation within a handheld tool. The intelligent instrument senses its own motion, distinguishes between voluntary and erroneous motion, and manipulates its tip to cancel the undesired component in real-time. The on-board sensing unit is made up of a magnetometer-aided allaccelerometer inertial measurement unit and sensor fusion is performed via a quaternion-based Kalman filtering. Tremor is modeled and filtered by an adaptive zero-phase notch filter. The intraocular shaft manipulator is a three DOF piezoelectric actuated mechanism driven by a feedforward controller with inverse rate-dependent hysteresis model. Laboratory experimental results of the system will be presented.
\end{abstract}

Keywords-Control system, inertial sensing, medical robotics, microsurgery, tremor.

\section{INTRODUCTION}

Humans have intrinsic limitations in manual positioning accuracy. These limitations are consequences of small involuntary movements that are inherent in normal hand motion. This manual imprecision hinders micromanipulation tasks, where the intended motion and the erroneous movements have the same order of magnitude, or in engineering terms: the signal-to-noise ratio is low. The influence it has on the performance of such tasks may range from significantly hampered, e.g. microsurgery [1] and cell manipulation in bio-tech industry, to moderate or slight degradation, e.g. consumer video cameras.

The most familiar type of erroneous movement affecting micromanipulation is hand tremor [2]. Tremor is defined as any involuntary, approximately rhythmic, and roughly sinusoidal movement [3]. Physiological tremor is a type of tremor that is inherent in the movement of healthy subjects. In ophthalmological microsurgery, the significant component of physiological tremor is found to be an oscillation at 8-12 Hz whose frequency is independent of the mechanical properties of the hand and arm [3]. The measured tool tip oscillation during vitreoretinal microsurgery can be as large as $50 \mu \mathrm{m}$ p-p in each of the principal axes [4]. It has also been reported that the vector magnitude of physiological tremor during the most delicate part of the procedure is measured to be $38 \mu \mathrm{m}$ rms [5].

These inherent limitations complicate many delicate surgical procedures, and make some types of intervention impossible. One of the common procedures in vitreoretinal microsurgery includes removal of membranes as thin as 20 $\mu \mathrm{m}$ from the retina. Another example is the treatment of retinal vein occlusions by injection of anticoagulants using intraocular cannulation [6]-[7]. This procedure holds the potential of curing a major cause of blindness, estimated to affect $1.6 \%$ of persons aged 49 years or older [8]. However, it is generally considered infeasible as it is practically impossible to insert a micropipette into a $\sim \varnothing 100 \mu \mathrm{m}$ retinal vein and deliver the drug without risking to tear the vessel apart. Other examples include procedures in neurological surgery and microvascular surgery.

The high level of manual accuracy demanded by microsurgery restricts the number of qualified surgeons. The fact that human hand stability deteriorates with age further exacerbates the situation. Even for microsurgeons in their prime years, fatigue, alcohol and caffeine consumption, and other factors affect the manual stability [9], [10]. Besides governing their sleep, and alcohol and caffeine consumptions prior to the surgery, some surgeons take beta blockers, which have been shown to have an attenuating effect [11]. There is some degree of consensus among vitreoretinal microsurgeons that instrument-tip positioning accuracy of $10 \mu \mathrm{m}$ is desired [12], a level that requires assistance from robotic technology.

Instead of going with more conventional robotic approaches [13], [14], we propose to implement tremor compensation in a completely handheld microsurgical instrument. The instrument is designed to sense its own motion, distinguish between intended and erroneous motion, and deflect its tip to cancel the undesired component in realtime. In comparison, this approach has the advantages of lower cost, less obtrusive to the surgeon, and has potentially fewer safety and liability issues, since the manipulator system has only a limited actuation stoke of a few hundred micrometers. Also, a failure of the system merely reduces it to a usual passive microsurgical tool, and will not cause any major disruption in the proceeding of the surgery. On the flip side, this active compensation approach requires the undesired motion component to be accurately estimated rather than just filtered out. This imposes technical challenges that form the subject matters of this paper. The following sections of the paper describe the theoretical and implementation issues of each of the sub-systems and laboratory experimental results.

\section{Micron SyStem OVERVIEW}

The intelligent active microsurgical instrument is named 'Micron'. The instrument weighs $<100 \mathrm{~g}$ and measures $180 \mathrm{~mm}$ in length, excluding the intraocular shaft. The diameter of the handle is $\varnothing 20 \mathrm{~mm}$ with $\varnothing 16 \mathrm{~mm}$ at the narrowest part of the contoured section to aid grasping. The back sensor suite measures $26 \mathrm{~mm} \times 26 \mathrm{~mm} \times 36 \mathrm{~mm}$. The instrument is designed to allow interchanging of disposable surgical needles and other tools with female Luer connectors as the intraocular shaft. 
The Micron system, as shown in Fig.1, is made up of three subsystems, the sensing system that senses the 6 DOF motion of the instrument, the filtering algorithms that model and estimate the erroneous motion component, and the intraocular shaft manipulator system that cancels the undesired motion component at the tool-tip. The overview of the Micron system is depicted in Fig. 2.

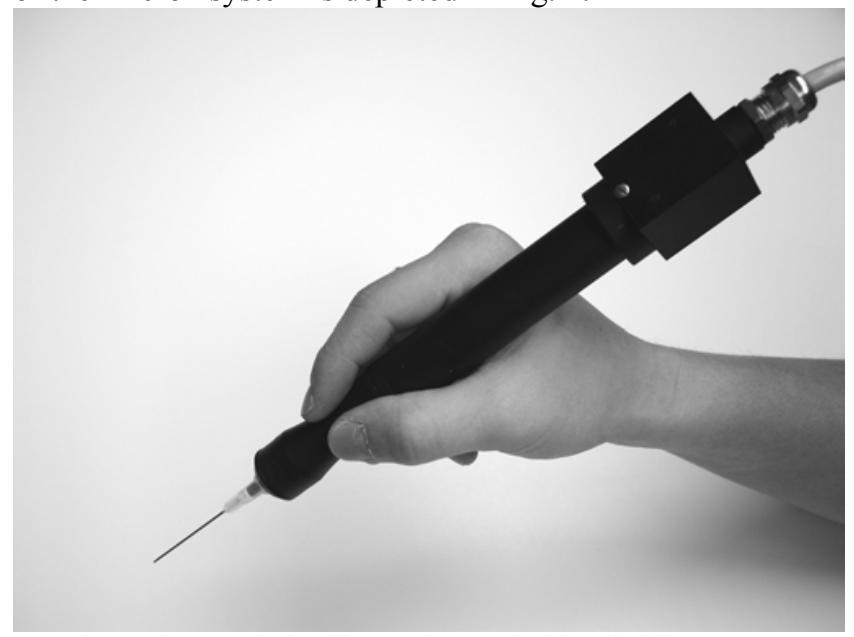

Fig. 1 Micron - the intelligent microsurgical handheld instrument.

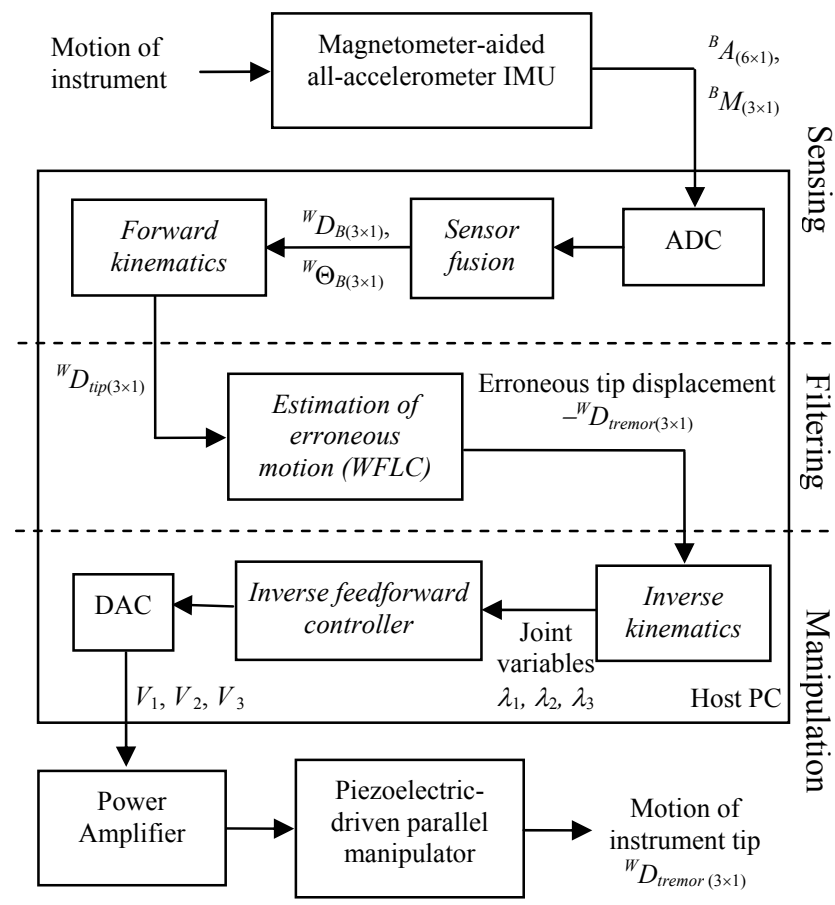

Fig. 2 Overview of the Micron system.

\section{Motion SENSING}

Instantaneous motion of the instrument is sensed by a new magnetometer-aided all-accelerometer inertial measurement unit (IMU) [15]. The sensing system consists of three dual-axis miniature accelerometers (Analog Devices
ADXL-203) and a three-axis magnetometer (Honeywell HMC-2003). The accelerometers are housed in two separate locations in the handheld device, as shown in Fig. 3. The sensor suite at the back end of the handle houses two dualaxis accelerometers in orthogonal orientations (one $X$-, one $Y$-, and two $Z$-sensing directions). One dual-axis accelerometer is located at the front end, measuring motion in $X$ and $Y$ directions.

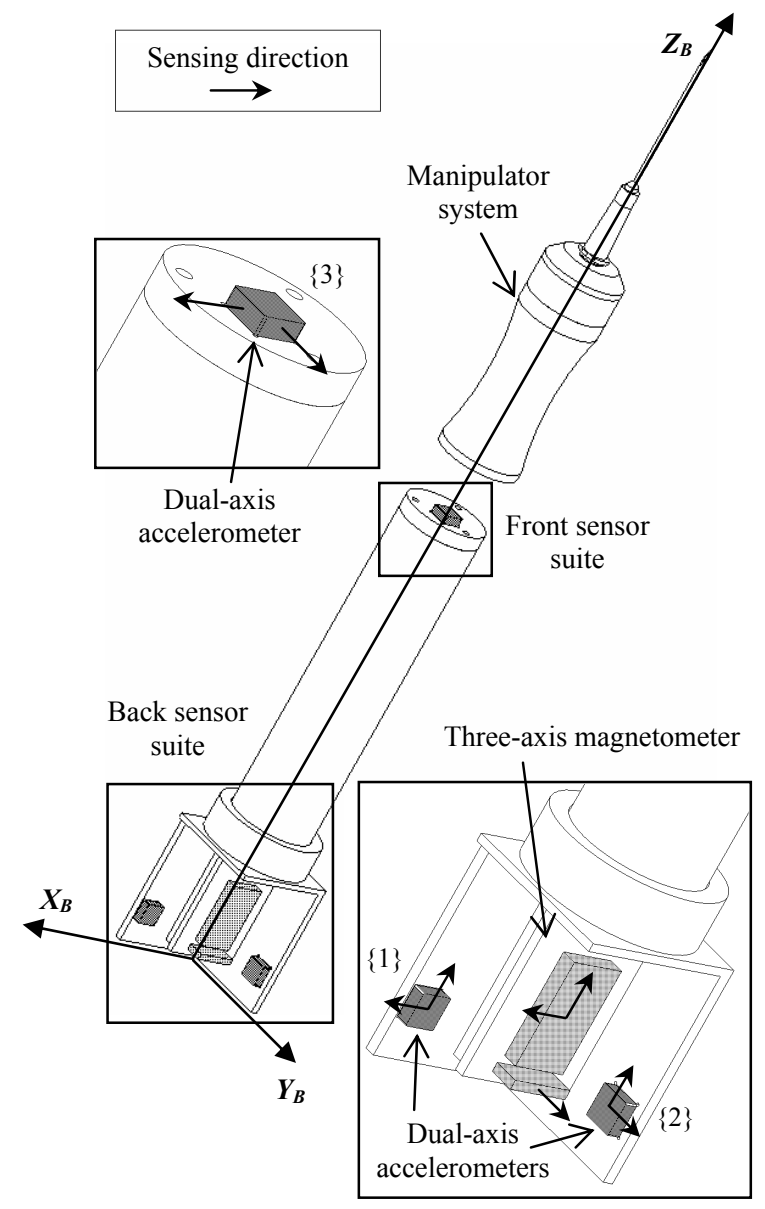

Fig. 3 Micron sensing system.

The redundancy in sensing provides two sources of orientation and position information. Angular motion information can be derived from the all-accelerometer IMU based on the principle of differential sensing kinematics. Taking a difference between outputs of accelerometers in the same sensing direction, we eliminate the translationinduced acceleration components, i.e. inertial acceleration at the body center of mass and gravity. Hence, the differential acceleration contains only the rotation-induced components, i.e. centripetal and tangential accelerations,

$$
A_{i j}=A_{j}-A_{i}=\Omega \times \Omega \times P_{i j}+\dot{\Omega} \times P_{i j},
$$

where $\Omega=\left[\begin{array}{lll}\omega_{x} & \omega_{y} & \omega_{z}\end{array}\right]^{T}$ is angular velocity vector and $P_{i j}, i, j$ $=1,2,3$, is the known position vector from $\{i\}$ to $\{j\}$. With the initial states well initialized, the angular acceleration vector $\dot{\Omega}$ can be expressed in terms of $\Omega$ and its time 
history. Thus, the angular velocity vector $\Omega$ may be readily solved with the three differential accelerations.

A second orientation sensing source is from the combination of the gravity and magnetic North vectors by the TRIAD algorithm [16]. It has been shown that the angular motion information derived from the differential sensing kinematics has very high sensing resolution but suffers from integration drift; on the other hand, the orientation obtained from the TRIAD algorithm is noisy but non-drifting. These two complementary sensing sources are fused via an augmented state quaternion-based Kalman filter to yield high quality sensing [16].

The sensing resolution of the translational motion can also be improved by up to a factor of $2^{1 / 2}$ by compounding the redundant acceleration sensing in each axis. On top of this, the improvement in the orientation estimation will result in a much complete removal of the gravity, which in turn has a great impact on the quality of the translation sensing since gravity is typically two orders of magnitude larger than hand accelerations.

\section{TREMOR MODELING AND FILTERING}

Estimation of tremor will be performed by a system based on the weighted-frequency Fourier linear combiner (WFLC) algorithm [17]. The WFLC is an adaptive algorithm that estimates tremor using a dynamic sinusoidal model, estimating its time-varying frequency, amplitude, and phase online. Active canceling of physiological tremor using this algorithm has been demonstrated using a 1-dof instrument prototype. In 25 tests on hand motion recorded from eye surgeons, this technique yielded average rms amplitude reduction of $69 \%$ in the $6-16 \mathrm{~Hz}$ band, and average rms error reduction of $30 \%$ with respect to an offline estimate of the tremor-free component of motion.

\section{INTRAOCULAR SHAFT MANIPULATOR}

The proposed design of the intraocular shaft manipulator is a three degrees-of-freedom parallel mechanism driven by piezoelectric actuators, as shown in Fig. 4. The intraocular shaft is attached to a standard Luer male connector fixed at the center of the three-legged rigid star. The three legs of the rigid star form the apexes of an equilateral triangle. The rigid star is screwed onto the flexistar, by a contact pin at each of its legs. The flexi-star is a flexible thin plate made of stainless steel spring steel. The three overhanging legs of the flexi-star is deflected by three stainless steel lever hinged $120^{\circ}$ apart near the front opening of the narrowed section of the handle that aids grasping. The three levers, which crisscross one another at different heights, serve as mechanical linkages that amplify the stroke of the piezoelectric actuators by 8 times, yielding a total stroke of $>100 \mu \mathrm{m}$ per axis. Three piezoelectric actuators are located on the three faces of the triangular column, and sandwiched between the base star and the levers pins. When voltage is applied to the piezoelectric stacks, they expand and rotate the levers which in turn push against the contact pins to move the star assembly and the intraocular shaft.

Piezoelectric emerges as the apparent choice of actuator because of its high actuation bandwidth. However, the highly nonlinear hysteretic behavior of the piezoelectric ceramic has made accurate and stable control of the actuator difficult, especially in applications tracking dynamic motion profiles such as tremor. To solve this problem, a ratedependent Prandtl-Ishlinskii hysteresis model is proposed, and the inverse of hysteresis model is implemented in an open-loop feedforward controller to linearize the piezoelectric hysteresis [18]. Experimental result has shown that the controller is capable of tracking a negated tremor profiles with rms tracking error of $0.6 \%$.

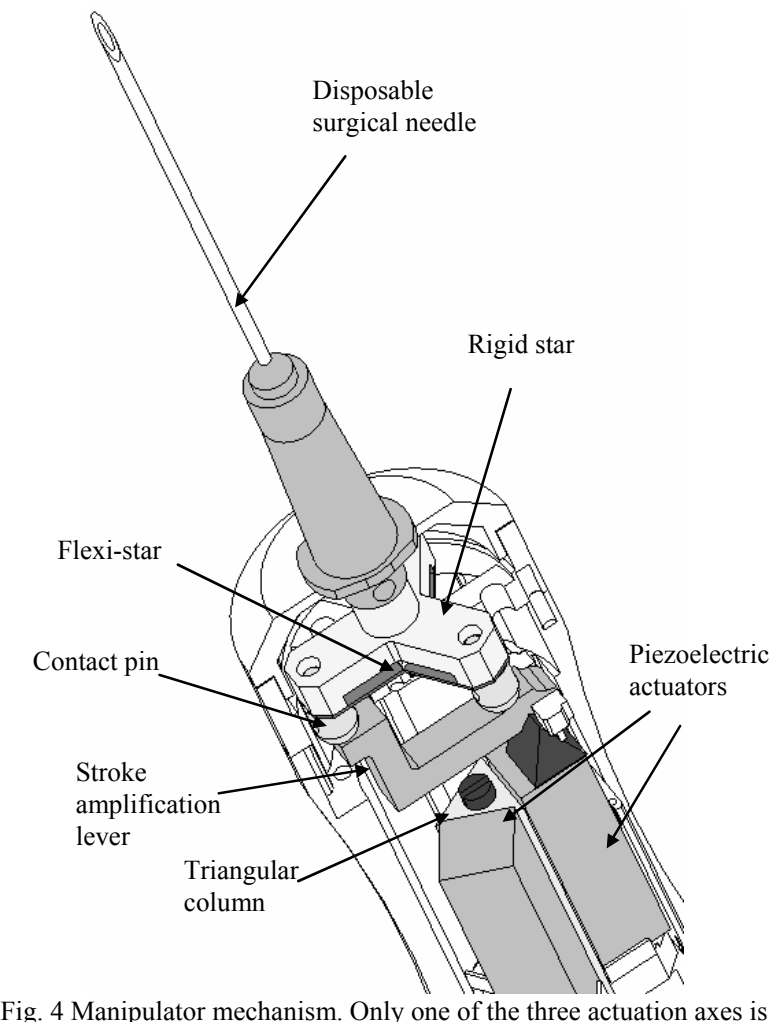

Fig. 4 Manipulator mechanism. Only one of the three actuation axes is shown in the illustration.

\section{MOTION CANCELING EXPERIMENT}

The experimental setup consists of two separate systems, the testbed oscillator and the optical motion tracking system.

The testbed generates oscillatory motion, simulating the hand tremor of the surgeon. The oscillating plate where Micron is mounted rests on a bearing-loaded linear slide. A spring-loaded driving shaft is attached to the back end of the plate. The shaft is driven by a DC servomotor at $9 \mathrm{~Hz}$, with amplitude of about $90 \mu \mathrm{m}$ p-p.

An optical motion tracking system [19], is used to measure the motion of the instrument tip. ASAP uses light- 
emitting diodes to illuminate the workspace. It measures instrument tip position in 3D using two $2 \mathrm{D}$ position sensitive detectors to sense the reflected light from a marker ball at the instrument tip.

Motion canceling tests involving one, two, or all three Cartesian coordinates (with respect to the instrument handle) can be performed by reorienting the direction of the testbed oscillation.

\section{RESULT}

Fig. 5 show the typical results of the $z$ axis of a 3D motion canceling test over a period of 1 second. The rms amplitude of the tip's motion generated by the testbed oscillator is $24.7 \mu \mathrm{m}$ at $9 \mathrm{~Hz}$ and the rms amplitude of an error compensated motion is $11.9 \mu \mathrm{m}$ or a reduction of $51.9 \%$ over a set of 10 runs.

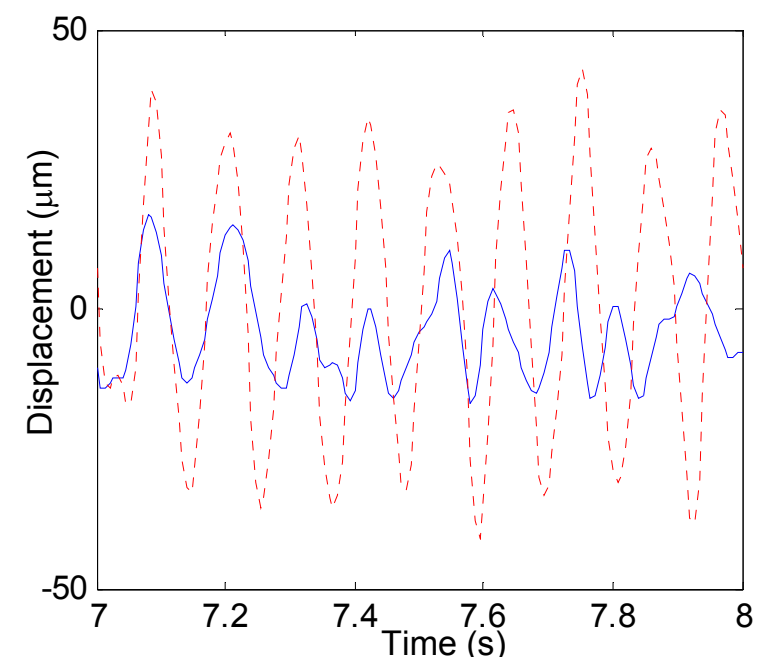

Fig 8 . Motion canceling result of Micron in $3 \mathrm{D}$ motion canceling test. The plot shows result in the $\mathrm{Z}$-axis. The dotted line depicts the uncompensated motion and the solid line depicts the compensated motion.

\section{DISCUSSION}

The Micron instrument reported in this paper is an improved version of the previous prototype. The motion canceling experiment has demonstrated the capability of Micron in performing real-time tremor compensation. More engineering fine tuning would definitely improve the performance of the integrated system to realize the full potential manifested by independent evaluation of each of the subsystems as reported in [15]-[18].

Future work of the instrument include mocked surgery evaluation using pig eyes, vitreoretinal microsurgery trials on live rabbits and dogs, and cell dissection experiments in biological laboratory.

\section{CONCLUSION}

The design and implementation of an active intelligent handheld microsurgical instrument that performs real-time tremor compensation has been presented. Laboratory testing has shown that the instrument successfully reduces simulated tremulous motion by $51.9 \%$.

\section{REFERENCES}

[1] M. Patkin, Ergonomics applied to the practice of microsurgery. Austr. N. Z. J. Surg. 47:320-239, 1977.

[2] R. C. Harwell and R. L. Ferguson, "Physiologic tremor and microsurgery," Microsurgery, vol. 4, pp. 187-192, 1983.

[3] R. J. Elble and W. C. Koller, Tremor. Baltimore: Johns Hopkins, 1990.

[4] I. W. Hunter, T. D. Doukoglou, S. R. Lafontaine, P. G. Charette, L. A. Jones, M. A. Sagar, G. D. Mallinson, and P. J. Hunter, "A teleoperated microsurgical robot and associated virtual environment for eye surgery," Presence, 2:265-280, 1993.

[5] S. P. N. Singh and C. N. Riviere, "Physiological tremor amplitude during retinal microsurgery," in Proc. IEEE 28th Annu. Northeast Bioeng. Conf., pp. 171-172, 2002.

[6] W. M. Tang and D.P. Han, "A study of surgical approaches to retinal vascular occlusions." Arch. Ophthalmol. 118:138-43, 2000.

[7] J. N. Weiss, "Treatment of central retinal vein occlusion by injection of tissue plasminogen activator into a retinal vein," Am. J. Ophthalmol. 126:142-144, 1998.

[8] P. Mitchell, W. Smith, and A. Chang, "Prevalence and associations of retinal vein occlusion in Australia: the Blue Mountains Eye Study," Arch. Ophthalmol., vol. 114, pp. 1243-1247, 1996.

[9] M. U. Humayun, R. S. Rader, D. J. Pieramici, C. C. Awh, E. de Juan, Jr., "Quantitative measurement of the effects of caffeine and propranolol on surgeon hand tremor," Arch. Ophthalmol., vol. 115, pp. 371-374, Mar. 1997.

[10] Lakie, M., Frymann, K., Villagra, F., Jakeman, P., "The effect of alcohol on physiological tremor," Experimental Physiology 79 (1994) 273-276

[11] M. J. Elman, J. Sugar, R. Fiscella, T. A. Deutsch, J. Noth, M. Nyberg, K. Packo, R. J. Anderson, "The effect of propranolol versus placebo on resident surgical performance," Trans. Am. Ophthalmol. Soc., vol. 96, pp. 283-291, 1998.

[12] S. Charles, Dexterity enhancement for surgery. In: R.H. Taylor et al. (eds.): Computer Integrated Surgery: Technology and Clinical Applications. MIT Press, Cambridge (1996) 467-471.

[13] H. Das, H. Zak, J. Johnson, J. Crouch, and D. Frambach, "Evaluation of a telerobotic system to assist surgeons in microsurgery," Computer Aided Surg., vol. 4, pp. 15-25, 1999.

[14] R. Taylor, P. Jensen, L. Whitcomb, A. Barnes, R. Kumar, D. Stoianovici, P. Gupta, Z. Wang, E. de Juan, and L. Kavoussi, "A steady-hand robotic system for microsurgical augmentation," in: C. Taylor, A. Colchester (eds.), Med. Image Computing \& ComputerAssisted Intervention - MICCAI'99, Springer, pp. 1031-1041.

[15] W. T. Ang, P. K. Khosla, and C. N. Riviere, "Design of AllAccelerometer Inertial Measurement Unit for Tremor Sensing in Hand-held Microsurgical Instrument", Proc. International Conference on Robotics and Automation, May 2003, pp.1781-1786.

[16] W. T. Ang, P. K. Khosla, and C. N. Riviere, "Kalman Filtering for Real-Time Orientation Tracking of Handheld Microsurgical Instrument," Submitted to. IEEE/RSJ Intl. Conf. Intelligent Robots and Systems, Oct 2004

[17] C. N. Riviere, R. S. Rader, and N. V. Thakor, "Adaptive canceling of physiological tremor for improved precision in microsurgery," IEEE Trans. Biomed. Eng., vol. 45, pp. 839-846, 1998.

[18] W. T. Ang, P. K. Khosla, and C. N. Riviere, "Inverse Feedforward Controller with Rate-dependent Hysteresis Model for Piezoelectric Actuators", Submitted to IEEE Trans Control Systems \& Tech.

[19] Hotraphinyo, L. F. and Riviere, C. N., "Precision measurement for microsurgical instrument evaluation", Proc. of the 23rd Annu. Conf. IEEE Eng. Med. Biol. Soc., Volume: 4 , 2001, pp. 3454 -3457 